\title{
Ganho genético com base no tamanho efetivo populacional de progênies de seringueira
}

\author{
Flávio Cese Arantes ${ }^{(1)}$, Paulo de Souza Gonçalves(2), Erivaldo José Scaloppi Junior ${ }^{(3)}$, \\ Mario Luiz Teixeira de Moraes $^{(4)}$ e Marcos Deon Vilela de Resende(5)
}

\begin{abstract}
(1)Universidade Estadual Paulista (Unesp), Faculdade de Ciências Agrárias e Veterinárias, Departamento de Ciências Exatas, Via de Acesso Prof. Paulo Donato Castellane, s/no , CEP 14884-900 Jaboticabal, SP. E-mail: flavionex@hotmail.com (2)Instituto Agronômico, Caixa Postal 28, CEP 13001-970 Campinas, SP. E-mail: paulog@iac.sp.gov.br ${ }^{(3)}$ Agência Paulista de Tecnologia dos Agronegócios, Pólo Regional Noroeste Paulista, Caixa Postal 61, CEP 15500-970 Votuporanga, SP. E-mail: scaloppi@apta.sp.gov.br (4)Unesp, Faculdade de Engenharia de Ilha Solteira, Departamento de Fitotecnia, Tecnologia dos Alimentos e Socioeconomia, Avenida Brasil, no 56, CEP $15385-000$ Ilha Solteira, SP. E-mail: teixeira@agr.feis.unesp.br ${ }^{(5)}$ Embrapa Florestas, Estrada da Ribeira, Km 111, Caixa Postal 319, CEP 83411-000 Colombo, PR. E-mail: deon@cnpf.embrapa.br
\end{abstract}

Resumo - O objetivo deste trabalho foi estimar os ganhos genéticos de um teste de progênies de seringueira para a produção de borracha seca e, com base no maior tamanho efetivo populacional e maior ganho genético, obter os melhores indivíduos. Foram utilizadas 30 progênies de meios-irmãos, provenientes de sementes de polinização mista - alogamia e autogamia - de testes clonais no Estado de São Paulo. Utilizou-se o delineamento experimental de blocos ao acaso, com 30 tratamentos (progênies), 3 repetições e parcelas lineares de 10 plantas, em um espaçamento de $3 \times 3 \mathrm{~m}$, o que totalizou 900 plantas úteis. Aos três anos, o perímetro, a $50 \mathrm{~cm}$ do solo (PA50), e a produção de borracha seca (PBS) foram avaliadas por meio do teste precoce de produção Hamaker Morris-Mann (HMM). As variáveis foram analisadas pelo método de modelo linear misto, via procedimento REML/BLUP, em progênies com sistema reprodutivo misto e taxa de autofecundação de $22 \%$. A identificação dos 20 melhores indivíduos quanto à PBS e ao PA50 proporcionou ganho genético de 67,96 e 16,48\%, respectivamente, e um coeficiente de endogamia de aproximadamente $2,82 \%$. O teste de progênies proporciona produção de sementes com melhor valor genético, grande variabilidade e baixa endogamia.

Termos para indexação: Hevea brasiliensis, índice multiefeitos, REML/BLUP, teste precoce HMM.

\section{Genetic gain based on effective population size of rubber tree progenies}

\begin{abstract}
The objective of this work was to evaluate genetic gains of a rubber tree progeny test for dry rubber yield and, based in the largest effective size and largest genetic gain, to get the best individuals. Thirty progenies of half-sib from seeds of mixed pollination - autogamy and outcrossing - clone tests from state of São Paulo were used. An experimental design in randomized block was used with 30 treatments (progenies), three replications, and plots of 10 plants, spaced $3 \times 3 \mathrm{~m}$, comprising a total of 900 useful plants. At three years old, girth growth at $50 \mathrm{~cm}$ of the ground (PA50) and dry rubber yield (PBS) were evaluated using the test Hamaker-Mann Morris (HMM). The variables were analyzed through the method of linear mixed model REML/BLUP in progenies that showed mixed mating system and self-pollination rate of $22 \%$. The identification of the 20 best individuals for PBS and PA50 provided a genetic gain of 67.96 and 16.48\%, respectively, and an inbreeding coefficient of approximately $2.82 \%$. The progeny test provides seed production with better breeding value, high genetic variability and low inbreeding.
\end{abstract}

Index terms: Hevea brasiliensis, multi-effects index, REML/BLUP, HMM test.

\section{Introdução}

Os testes de progênies, instrumentos importantes para o trabalho do melhorista, são usados na estimativa de parâmetros genéticos e seleção de indivíduos, quando se quer avaliar a magnitude e a natureza da variância genética disponível, a fim de quantificar e maximizar os ganhos genéticos, com uso de procedimento de seleção adequado. Os caracteres normalmente avaliados nos testes de progênie com a seringueira são altura, perímetro do caule e produção de látex. A seleção geralmente baseia-se na produção de látex (Costa et al., 2008).

A seleção com base em teste de progênies é sempre mais eficiente do que a realizada como base apenas no fenótipo das plantas individuais, pela avaliação não só

Pesq. agropec. bras., Brasília, v.45, n.12, p.1419-1424, dez. 2010 
dos indivíduos a serem selecionados como também dos seus descendentes (Paiva et al., 2002). Segundo Kageyama \& Vencovsky (1983), a seleção individual intensa para um caráter de alta herdabilidade deve ser vista com cautela, pois essa prática poderá provocar eliminação ou perda precoce de alelos responsáveis por outros caracteres. Os riscos de seleção individual intensa, no melhoramento genético, somente podem ser reduzidos se, entre outros fatores, o tamanho efetivo da população for mantido alto.

Aestimação de componentes de variância com base na máxima verossimilhança restrita (REML) e a predição linear não viciada (BLUP) de valores genéticos têm sido adotadas no melhoramento de espécies perenes e anuais (Souza et al., 2000; Paiva et al., 2002; Eagles \& Moody, 2004; Missio et al., 2004, 2005; Bauer et al., 2006; Farias Neto et al., 2009; Pedrozo et al., 2009; Verardi et al., 2009; Viana et al., 2010a, 2010b). Mediante pesos de ponderação do indivíduo e da família, é possível selecionar indivíduos com características favoráveis dentro de famílias de desempenho intermediário ou, ainda, indivíduos de desempenho intermediário dentro de famílias superiores. No caso de plantas perenes, uma seleção combinada aperfeiçoada deve incluir também o efeito da parcela. Esse método de seleção é denominado Índice Multiefeitos (Resende \& Higa, 1994).

O ciclo de melhoramento da seringueira ocorre em três etapas. A primeira consiste em avaliações por meio de testes precoces de produção, vigor e tolerância a doenças. Os ortetes são selecionados e clonados para serem testados em experimentos de avaliação de clones em pequena escala. Nessa segunda etapa do ciclo de seleção, após os dois primeiros anos de sangria, os clones promissores são multiplicados e passam a ser avaliados em grande escala, ou ensaios regionais. Na última etapa, são gastos geralmente de 12 a 15 anos, até que se possa recomendar um clone para plantio em grande escala. Portanto, são necessários cerca de 30 anos para completar o ciclo de melhoramento (Jain \& Priyadarshan, 2009).

Testes precoces de seleção têm revelado genótipos de seringueiras cada vez mais produtivos, o que acarreta, muitas vezes, diminuição da variabilidade genética e, consequentemente, do tamanho efetivo da população (Tan \& Subramanian, 1976).

Portanto, quanto maior a variabilidade genética, maior a possibilidade de ganho de seleção para o caráter desejado, e consequentemente o teste de progênie é uma das mais importantes etapas da seleção.
O objetivo deste trabalho foi estimar os ganhos genéticos de um teste de progênies de seringueira para a produção de borracha seca e, com base no maior tamanho efetivo populacional e maior ganho genético, obter os melhores indivíduos.

\section{Material e Métodos}

O experimento foi instalado no Município de Selvíria, MS, com localização geográfica aproximada da área do experimento na latitude de $20^{\circ} 20^{\prime} \mathrm{S}$, $51^{\circ} 23^{\prime} \mathrm{W}$ e altitude de $370 \mathrm{~m}$. O relevo é caracterizado por ser moderadamente plano e ondulado. O clima do local é do tipo Aw, pela classificação de Köppen, com temperatura média anual de $24,5^{\circ} \mathrm{C}$, precipitação média anual de $1.232 \mathrm{~mm}$, umidade média anual de $64,8 \%$ e insolação média de 7,3 horas por dia (Hernandez et al., 1995). O solo local foi classificado de acordo com o Sistema Brasileiro de Classificação de Solos (Santos et al., 2006), como Latossolo Vermelho distrófico (LVd) típico argiloso, A moderado, hipidistrófico, álico, caulinítico, férrico, compactado, muito profundo, moderadamente ácido.

Foram utilizadas 30 progênies de meios-irmãos provenientes de sementes de polinização mista (alogamia e autogamia), obtidas de 30 clones no Estado de São Paulo: 1-12-56-77, 64B 850, Fx (2261 e 3864), GT1, IAC $(15,301,307,311,35,40,41$ e 44), IAN 873, IRCA 111, MT 45, PB (217, 235, 252, 260, 28/59 e 330), Pind 595/89, PR 255 e 261, RO/I (35 e 110) e RRIM (600, 606 e 701), os quais se encontram na Agência Paulista de Tecnologia dos Agronegócios (APTA), Pólo Regional do Noroeste Paulista, Votuporanga.

As sementes foram colocadas para germinar em sacos de polietileno, e as progênies foram transportadas para o local definitivo, quando possuíam dois lançamentos foliares. Foi utilizado o delineamento de blocos ao acaso, compostos por 30 tratamentos (progênies), três repetições e parcelas lineares de 10 plantas, em um espaçamento de $3 \times 3 \mathrm{~m}$, que totalizou 900 plantas úteis.

Aos três anos de idade, as progênies foram avaliadas quanto ao perímetro, à altura de $50 \mathrm{~cm}$ do solo (PA50), com fita métrica. A produção de borracha seca (PBS), em gramas, foi obtida pelo teste Hamaker Morris-Mann (HMM), modificado (Tan \& Subramanian, 1976) para plantas de três anos de idade, tendo-se utilizado a produção de borracha seca de dez cortes por planta. $\mathrm{O}$ painel de sangria foi aberto a $30 \mathrm{~cm}$ do solo, pelo sistema $1 / 2 \mathrm{~S} d / 2$ ET $2,5 \%$, no total de 15 cortes, com 
descarte das cinco primeiras amostras que correspondem à fase de amansamento do painel. A nomenclatura $1 / 2 \mathrm{~S}$ corresponde ao corte em meio espiral, e a nomenclatura $\mathrm{d} / 2$ expressa o intervalo entre sangrias, uma sangria a cada dois dias, e $2,5 \%$ de etefon (ET) corresponde à concentração do produto comercial, aplicado a $1 \mathrm{~mL}$ por planta.

As variáveis foram analisadas com uso do método de modelo linear misto via procedimento máxima verossimilhança restrita/melhor preditor linear não viciado (REML/BLUP) em progênies de polinização aberta, com sistema reprodutivo misto, taxa de autofecundação (s) de $22 \%$, percentagem com base em trabalhos de Costa et al. (2000a) e Furlani et al. (2005), com delineamento experimental de blocos ao acaso e com várias plantas por parcela, segundo Resende (2002a). Utilizou-se o modelo estatístico $\mathrm{y}=\mathrm{Xr}+\mathrm{Za}+\mathrm{Wc}+\varepsilon$, em que: y é o vetor de dados, r é $o$ vetor dos efeitos de repetição, assumidos como fixos, somados à média geral, a é o vetor dos efeitos genéticos aditivos individuais, assumidos como aleatórios, c é o vetor dos efeitos de parcela, $\varepsilon$ é o vetor de erros ou resíduos aleatórios. As letras maiúsculas representam as matrizes de incidência para os efeitos.

Com base nos componentes de variância associados aos efeitos, foram estimados os seguintes parâmetros:

$$
\hat{\mathrm{h}}_{\mathrm{g}}^{2}=\left[4 \hat{\sigma}_{\mathrm{g}}^{2} /(1+\mathrm{s})^{2}\right] /\left(\hat{\sigma}_{\mathrm{g}}^{2}+\hat{\sigma}_{\mathrm{c}}^{2}+\hat{\sigma}_{\mathrm{e}}^{2}\right),
$$

herdabilidade individual no sentido restrito, com taxa de autofecundação s de $22 \%$;

$$
\hat{\mathrm{c}}^{2}=\left(\hat{\sigma}_{\mathrm{c}}^{2}\right) /\left(\hat{\sigma}_{\mathrm{g}}^{2}+\hat{\sigma}_{\mathrm{c}}^{2}+\hat{\sigma}_{\mathrm{e}}^{2}\right),
$$

coeficiente de determinação dos efeitos de parcela;

$$
\hat{\mathrm{h}}_{\mathrm{mp}}^{2}=\left(\hat{\sigma}_{\mathrm{g}}^{2}\right) /\left[\hat{\sigma}_{\mathrm{g}}^{2}+\hat{\sigma}_{\mathrm{c}}^{2} / \mathrm{b}+\hat{\sigma}_{\mathrm{e}}^{2} /(\mathrm{nb})\right],
$$

herdabilidade da média de progênies, quando se considera a sobrevivência completa;

$$
\hat{\mathrm{r}}_{\mathrm{a} a}^{2}=\left(\hat{\mathrm{h}}_{\mathrm{mp}}^{2}\right)^{0,5},
$$

acurácia da seleção de progênies, quando se considera a sobrevivência completa;

$$
\hat{\mathrm{h}}_{\mathrm{ad}}^{2}=\left\{1-\left[(1+\mathrm{s})^{2} / 4\right]\right\}\left[4 /(1+\mathrm{s})^{2}\right] \hat{\sigma}_{\mathrm{g}}^{2} / \hat{\sigma}_{\mathrm{e}}^{2},
$$

herdabilidade aditiva dentro de parcela;

$$
\mathrm{CV}_{\mathrm{g}}(\%)=100\left(\hat{\sigma}_{\mathrm{g}}^{2}\right)^{0,5} / \mu
$$

coeficiente de variação genética;

$$
\mathrm{CV}_{\mathrm{e}}(\%)=100\left(\hat{\sigma}_{\mathrm{e}}^{2}\right)^{0,5} / \mu,
$$

coeficiente de variação ambiental dentro da parcela. O termo,

$$
\hat{\sigma}_{\mathrm{f}}^{2}=\hat{\sigma}_{\mathrm{g}}^{2}+\hat{\sigma}_{\mathrm{c}}^{2}+\hat{\sigma}_{\mathrm{e}}^{2},
$$

é a estimativa da variância fenotípica, em que: $\hat{\sigma}_{\mathrm{g}}^{2}$, estimativa da variância genética entre famílias, cujos componentes da variância genética aditiva e variância genética de dominância dependem da taxa de autofecundação. $\hat{\sigma}_{c}^{2}$, estimativa da variância entre parcelas. $\hat{\sigma}_{\mathrm{e}}^{2}$, estimativa da variância residual dentro de parcelas (ambiental + não aditiva).

As estimativas dos parâmetros genéticos foram obtidas pelo procedimento Selegen REML/BLUP a partir de iterações nas equações de modelo misto (Resende, 2002b).

O tamanho efetivo populacional $\left(\mathrm{N}_{\mathrm{e}}\right)$ foi obtido com base em Resende (2002a):

$$
\mathrm{N}_{\mathrm{e}}=\left(4 \mathrm{~N}_{\mathrm{f}} \overline{\mathrm{k}}_{\mathrm{f}}\right) /\left[\overline{\mathrm{k}}_{\mathrm{f}}+3+\left(\sigma_{\mathrm{kf}}^{2} / \overline{\mathrm{k}}_{\mathrm{f}}\right)\right],
$$

em que $\overline{\mathrm{k}}_{\mathrm{f}}$ é o número médio de indivíduos selecionados por matriz; $\sigma_{\mathrm{kf}}^{2}$ é a variância do número de indivíduos selecionados por matriz; $\mathrm{N}_{\mathrm{f}}$ é o número de matrizes selecionadas.

Com base na classificação de indivíduos, foi realizada a otimização da seleção em razão do tamanho efetivo da população com restrição no número máximo de indivíduos selecionados por progênie.

\section{Resultados e Discussão}

$\mathrm{O}$ coeficiente de variação genética $\left(\mathrm{CV}_{\mathrm{g}}\right)$, que expressa, em percentagem da média geral, a quantidade de variação genética existente, apresentou valor moderado para PBS e relativamente baixo para PA50, o que indica que a população pode ser considerada apropriada para o programa de melhoramento genético (Tabela 1). O valor do $\mathrm{CV}_{\mathrm{g}}$ para $\mathrm{PBS}$ corrobora estudos desenvolvidos em Votuporanga, SP (Moreti et al., 1994; Boock et al., 1995; Gonçalves et al., 1998a, 2005, 2009; Costa et al., 2000a, 2008), em Jaú, SP (Gonçalves et al., 1998b) e em Pindorama, SP (Gonçalves et al., 2004). O valor do $\mathrm{CV}_{\mathrm{g}}$ para PA50 corrobora os resultados obtidos por Gonçalves et al. (2005, 2009), em Votuporanga, SP, e Gonçalves et al. 
(1999), em Pindorama, SP. Tal variabilidade genética, associada aos baixos valores do coeficiente de variação experimental $\left(\mathrm{CV}_{\mathrm{e}}\right)$, propiciou herdabilidades individuais $\left(\hat{\mathrm{h}}_{\mathrm{g}}^{2}\right)$ significativas e altas. Esses valores representam uma situação muito favorável para a seleção.

A acurácia $\left(\hat{r}_{\hat{a} a}\right)$ ou correlação entre os valores genéticos preditos e os verdadeiros foi alta em ambos os caracteres. Segundo Resende (2002a), a acurácia é uma medida que está associada à precisão na seleção e é o principal componente do progresso genético que se pode alterar para maximizar o ganho genético.

O coeficiente de determinação dos efeitos de parcela $\left(\hat{c}^{2}\right)$ foi baixo, o que é indicação de que o delineamento experimental empregado foi adequado, pois permaneceu a homogeneidade ambiental dentro de blocos.

Os efeitos da parcela não foram significativos para os caracteres avaliados, o que significa que há homogeneidade entre os blocos (Tabela 2). O efeito de progênie foi significativo para ambos os caracteres, o que indica diferença significativa na produção e no perímetro entre as progênies avaliadas.

Para o caráter PA50, em razão de a restrição ter admitido no máximo dois indivíduos selecionados

Tabela 1. Estimativas de parâmetros genéticos para o caráter perímetro $(\mathrm{cm})$ à altura de $50 \mathrm{~cm}$ (PA50) e produção de borracha seca (PBS) em $g$ por planta em seringueira, aos três anos de idade, em um teste de progênies localizado em Selvíria, MS.

\begin{tabular}{lrr}
\hline Parâmetros & \multicolumn{1}{c}{ PA50 } & \multicolumn{1}{c}{ PBS } \\
\hline$\hat{\sigma}_{\mathrm{g}}^{2}$ & 4,8911 & 574,7824 \\
$\hat{\sigma}_{\mathrm{c}}^{2}$ & 0,0187 & 9,1358 \\
$\hat{\sigma}_{\mathrm{e}}^{2}$ & 17,8120 & 1441,1838 \\
$\hat{\sigma}_{\mathrm{f}}^{2}$ & 22,7218 & 2025,1020 \\
$\hat{\mathrm{h}}_{\mathrm{g}}^{2}$ & $0,58 \pm 0,19$ & $0,76 \pm 0,22$ \\
$\hat{\mathrm{c}}^{2}$ & 0,0008 & 0,0045 \\
$\hat{\mathrm{h}}_{\mathrm{mp}}^{2}$ & 0,9974 & 0,9938 \\
$\hat{\mathrm{r}}_{\mathrm{a} \mathrm{a}}$ & 0,9987 & 0,9969 \\
$\hat{\mathrm{h}}_{\mathrm{ad}}^{2}$ & 0,4634 & 0,6730 \\
$\mathrm{CV} \mathrm{V}_{\mathrm{g}}(\%)$ & 7,8730 & 25,3094 \\
$\mathrm{CV}_{\mathrm{e}}(\%)$ & 15,0249 & 40,0764 \\
\hline
\end{tabular}

Pesq. agropec. bras., Brasília, v.45, n.12, p.1419-1424, dez. 2010 por progênie, o ganho genético equivaleu a $4,62 \mathrm{~cm}$ e a média da população melhorada será de $32,71 \mathrm{~cm}$, ou seja, o ganho será de 16,48\% (Tabela 3). No entanto, Costa et al. (2008) obtiveram ganho genético negativo para o caráter perímetro $(-5,5 \%)$ em um teste de progênies com a mesma idade. Pode-se inferir que o teste de progênie avaliado neste trabalho serve como indicador de mudas de alto vigor. O coeficiente de endogamia ou de endocruzamento, associado às

Tabela 2. Análise de deviance para o caráter produção de borracha seca (PBS) e perímetro à altura de $50 \mathrm{~cm}$ (PA50) de seringueira, em Selvíria, $\mathrm{MS}^{(1)}$.

\begin{tabular}{lccccc}
\hline Efeito & \multicolumn{2}{c}{ PBS } & \multicolumn{2}{c}{ PA50 } \\
\cline { 2 - 3 } & Deviance & $\mathrm{X}^{2}$ & & Deviance & $\mathrm{X}^{2}$ \\
\cline { 5 - 6 } Progênie & 2947,07 & $60,49^{* *}$ & & 634,93 & $37,2^{* *}$ \\
Parcela & 2886,72 & $0,14^{\text {ns }}$ & & 597,73 & $0,0^{\text {ns }}$ \\
Modelo completo & 2886,58 & - & & 597,73 & - \\
\hline
\end{tabular}

(1) $\mathrm{X}^{2}$, qui-quadrado tabelado: 3,84 e 6,63 para a probabilidade de 5 e $1 \%$, respectivamente; ${ }^{\text {ns }}$ Não significativo a $5 \%$ de probabilidade. $* *$ Significativo a $1 \%$ de probabilidade.

Tabela 3. Relação dos 20 melhores indivíduos de seringueira para o caráter perímetro à altura de $50 \mathrm{~cm}$, com base nos efeitos aditivos (a) e valores genéticos aditivos $(\mu+a)$, com restrição ao tamanho efetivo populacional $(\mathrm{Ne})$ em um teste de progênies aos três anos de idade, localizado em Selvíria, $\mathrm{MS}^{(1)}$.

\begin{tabular}{clccccccc}
\hline Bloco & Progênie & Ortete & $\mathrm{f}$ & $\mathrm{a}$ & $\mu+\mathrm{a}$ & $\mathrm{GG}$ & $\mu$ melhorada & $\mathrm{Ne}$ \\
\hline 1 & IAC 41 & 21 & 41,10 & 7,97 & 36,06 & 7,97 & 36,06 & 1,00 \\
3 & IAC 307 & 890 & 40,50 & 5,71 & 33,80 & 6,84 & 34,93 & 2,00 \\
2 & IAC 41 & 516 & 39,10 & 5,61 & 33,70 & 6,43 & 34,52 & 2,48 \\
3 & IAC 40 & 801 & 41,00 & 5,52 & 33,62 & 6,20 & 34,30 & 3,49 \\
1 & RRIM 600 & 3 & 35,40 & 5,18 & 33,27 & 6,00 & 34,09 & 4,49 \\
2 & IAN 873 & 406 & 35,80 & 4,89 & 32,98 & 5,82 & 33,91 & 5,50 \\
2 & IAN 873 & 408 & 35,70 & 4,85 & 32,94 & 5,68 & 33,77 & 6,07 \\
3 & PB 28/59 & 870 & 38,90 & 4,69 & 32,78 & 5,55 & 33,64 & 7,06 \\
3 & PB 252 & 733 & 39,60 & 4,53 & 32,62 & 5,44 & 33,53 & 8,05 \\
1 & PR 261 & 220 & 34,50 & 4,48 & 32,57 & 5,34 & 33,43 & 9,05 \\
3 & IAC 15 & 695 & 38,00 & 4,39 & 32,48 & 5,26 & 33,35 & 10,04 \\
1 & PB 330 & 246 & 33,50 & 4,17 & 32,26 & 5,17 & 33,26 & 11,04 \\
1 & IRCA 111 & 82 & 32,60 & 4,15 & 32,24 & 5,09 & 33,18 & 12,04 \\
2 & IAC 301 & 507 & 37,20 & 4,03 & 32,12 & 5,01 & 33,10 & 13,03 \\
1 & Fx 3864 & 256 & 32,50 & 3,79 & 31,89 & 4,93 & 33,02 & 14,03 \\
1 & IAC 35 & 45 & 34,10 & 3,77 & 31,86 & 4,86 & 32,95 & 15,03 \\
2 & IRCA 111 & 470 & 34,70 & 3,68 & 31,77 & 4,79 & 32,88 & 15,58 \\
1 & GT1 & 236 & 32,80 & 3,67 & 31,76 & 4,73 & 32,82 & 16,58 \\
2 & PR 255 & 315 & 36,00 & 3,62 & 31,71 & 4,67 & 32,76 & 17,57 \\
1 & Fx 3864 & 257 & 32,10 & 3,61 & 31,70 & 4,62 & 32,71 & 18,14 \\
\hline (1)f, valor fenotípico observado; $\mu$ média geral = 28,09 cm; GG, ganho \\
genético em cm. & & & & & & &
\end{tabular}


sementes produzidas para o caráter PA50 no pomar, é de $\mathrm{F}=[1 /(2 \mathrm{Ne})] 100=2,76 \%$.

Para o caráter PBS (g por planta), como a restrição admitiu no máximo dois indivíduos selecionados por progênie, o ganho genético equivaleu a $64,38 \mathrm{~g}$ por planta e a média da população melhorada foi de 159,10 g por planta, ou seja, ganho de $67,96 \%$ (Tabela 4). Costa et al. (2000b, 2008) obtiveram um ganho genético de 18,36 e 16,2\%, respectivamente, para a PBS. No entanto, Costa et al. (2000a) obtiveram ganhos genéticos de 28,7 a $218,7 \%$ para a PBS e concluíram que superestimativas consideráveis de ganhos genéticos ocorrem na seleção individual combinada e índice multiefeitos, quando não se considera o sistema reprodutivo misto para a seringueira. A seleção com base no índice multiefeitos maximiza o progresso genético e deve ser utilizada.

O coeficiente de endogamia ou de endocruzamento, associado às sementes produzidas para PBS no pomar, é de $\mathrm{F}=[1 /(2 \mathrm{Ne})] 100=2,88 \%$. O coeficiente de endogamia foi semelhante nas duas variáveis analisadas.

Tabela 4. Relação dos 20 melhores indivíduos de seringueira para o caráter produção de borracha seca (PBS) em $g$ por planta, com base nos efeitos aditivos (a) e valores genéticos aditivos $(\mu+a)$, com restrição ao tamanho efetivo populacional $(\mathrm{Ne})$ em um teste de progênies aos três anos de idade, localizado em Selvíria, $\mathrm{MS}^{(1)}$.

\begin{tabular}{clccccccc}
\hline Bloco & Progênie & Ortete & $\mathrm{f}$ & $\mathrm{a}$ & $\mu+\mathrm{a}$ & $\mathrm{GG}$ & $\mu$ melhorada & $\mathrm{Ne}$ \\
\hline 3 & PB 28/59 & 870 & 223,60 & 87,13 & 181,85 & 87,13 & 181,85 & 1,00 \\
3 & RRIM 701 & 820 & 230,30 & 86,14 & 180,86 & 86,63 & 181,36 & 2,00 \\
2 & IAN 873 & 408 & 206,20 & 79,55 & 174,28 & 84,27 & 179,00 & 3,00 \\
1 & GT1 & 238 & 185,80 & 78,09 & 172,82 & 82,73 & 177,45 & 4,00 \\
1 & PR 261 & 220 & 187,70 & 75,44 & 170,16 & 81,27 & 175,99 & 5,00 \\
3 & IAC 40 & 808 & 216,50 & 74,35 & 169,08 & 80,12 & 174,84 & 6,00 \\
2 & PB 28/59 & 479 & 186,30 & 73,99 & 168,72 & 79,24 & 173,97 & 6,50 \\
1 & RRIM 606 & 96 & 178,00 & 68,91 & 163,63 & 77,95 & 172,67 & 7,50 \\
3 & IAC 40 & 804 & 203,80 & 67,00 & 161,72 & 76,73 & 171,46 & 8,05 \\
1 & GT1 & 240 & 168,20 & 66,81 & 161,54 & 75,74 & 170,47 & 8,64 \\
2 & IAC 41 & 517 & 169,80 & 60,50 & 155,23 & 74,35 & 169,08 & 9,63 \\
3 & IAC 41 & 874 & 186,30 & 59,99 & 154,72 & 73,16 & 167,88 & 10,23 \\
1 & RRIM 600 & 6 & 174,00 & 58,52 & 153,25 & 72,03 & 166,76 & 11,21 \\
3 & PB 252 & 739 & 189,10 & 55,39 & 150,11 & 70,84 & 165,57 & 12,20 \\
2 & RRIM 606 & 443 & 157,40 & 55,19 & 149,92 & 69,80 & 164,53 & 12,81 \\
1 & PB 217 & 229 & 160,80 & 53,61 & 148,34 & 68,79 & 163,51 & 13,79 \\
2 & PR 255 & 316 & 156,90 & 49,82 & 144,55 & 67,67 & 162,40 & 14,77 \\
3 & IAC 15 & 691 & 175,70 & 46,14 & 140,87 & 66,48 & 161,20 & 15,76 \\
2 & PR 255 & 315 & 148,50 & 45,60 & 140,32 & 65,38 & 160,10 & 16,36 \\
1 & IAC 301 & 147 & 154,30 & 45,37 & 140,10 & 64,38 & 159,10 & 17,34 \\
\hline
\end{tabular}

(1)f, valor fenotípico observado; $\mu$, média geral $=94,73 \mathrm{~g} \mathrm{planta}^{-1}$; GG: ganho genético em $\mathrm{g}$ por planta.
Das 20 progênies selecionadas para a $\mathrm{PBS}, 75 \%$ são semelhantes às selecionadas para PA50, as quais são oriundas dos clones PB (28/59 e 252), IAN 873, GT 1, PR (255 e 261), IAC (40, 41, 15 e 301) e RRIM 600.

\section{Conclusões}

1. O método de otimização da seleção de seringueira, com base no ganho genético e no tamanho efetivo populacional, proporciona a formação de pomar de produção de sementes com genótipos superiores e baixa endogamia.

2. Há ganho genético de produção de borracha seca na população melhorada de seringueira.

3. A população selecionada de seringueira pode ser usada na conservação genética da espécie, por conter grande variabilidade e baixa endogamia.

\section{Agradecimentos}

À Fundação de Amparo à Pesquisa do Estado de São Paulo, pelo apoio financeiro; e aos funcionários Alonso Ângelo da Silva, Manoel Fernando Rocha Bonfin e José Cambuim, da Fazenda de Ensino e Pesquisa da Universidade Estadual Paulista, pela ajuda na condução do experimento de campo e pela coleta de dados.

\section{Referências}

BAUER, A.M.; REETZ, T.C.; LÉON, J. Estimation of breeding values of inbred lines using best linear unbiased prediction (BLUP) and genetic similarities. Crop Science, v.46, 2685-2691, 2006.

BOOCK, M.V.; GONÇALVES, P.S.; BORTOLETTO, N.; MARTINS, A.L.M. Herdabilidade, variabilidade genética e ganhos genéticos para produção e caracteres morfológicos em progênies jovens de seringueira. Pesquisa Agropecuária Brasileira, v.30, p.673-681, 1995.

COSTA, R.B. da; RESENDE, M.D.V. de; ARAUJO, A.J. de; GONÇALVES, P. de S.; HIGA, A.R. Selection and genetic gain in rubber tree (Hevea) populations using a mixed mating system. Genetics and Molecular Biology, v.23, p.671-679, 2000a.

COSTA, R.B. da; RESENDE, M.D.V. de; ARAUJO, A.J. de; GONÇALVES, P. de S.; SILVA, M.A. Maximization of genetic gain in rubber tree (Hevea) breeding with effective size restriction. Genetics and Molecular Biology, v.23, p.457-462, 2000b.

COSTA, R.B. da; RESENDE, M.D.V. de; GONÇALVES, P. de S.; OLIVEIRA, L.C.S. de; ÍTAVO, L.C.V.; ROA, R.A.R. Seleção simultânea para porte reduzido e alta produção de látex em seringueira. Bragantia, v.67, p.649-654, 2008. 
EAGLES, H.A.; MOODY, D.B. Using unbalanced data from a barley breeding program to estimate gene effects: the $\mathrm{Ha}$, $\mathrm{Ha} 4$, and $s d w 1$ genes. Australian Journal of Agricultural Research, v.55, 379-387, 2004.

FARIAS NETO, J.T. de; LINS, P.M.P.; RESENDE, M.D.V. de; MULLER,A.A. Seleção genética em progênies híbridas de coqueiro. Revista Brasileira de Fruticultura, v.31, p.190-196, 2009.

FURLANI, R.C.M.; MORAES, C.M.B. de; MORAES, M.L.T. de; PAIVA, J.R. de; SEBBENN, A.M. Mating system in a Hevea brasiliensis population by isozyme loci. Crop Breeding and Applied Biotechnology, v.5, p.402-409, 2005.

GONÇALVES, P. de S.; AGUIAR, A.T. de E.; COSTA, R.B. da; GONÇALVES, E.C.P.; SCALOPPI JUNIOR, E.J.; FERRAZ BRANCO, R.B. Genetic variation and realized genetic gain from rubber tree improvement. Sciencia Agricola, v.66, p.44-51, 2009.

GONÇALVES, P. de S.; BORTOLETTO, N.; MARTINS, A.L.M.; GOTTARDI, M.V.C.; ORTOLANI, A.A. Variação genética da produção de látex e incremento do caule em progênies de seringueira. Pesquisa Agropecuária Brasileira, v.33, p.321-330, 1998a.

GONÇALVES, P. de S.; BORTOLETTO, N.; SANTOS, W.R. dos; ORTOLANI, A.A.; GOTTARDI, M.V.C.; MARTINS, A.L.M. Avaliação genética de progênies em meios-irmãos de seringueira em diferentes regiões do Estado de São Paulo. Pesquisa Agropecuária Brasileira, v.33, p.1085-1095, 1998 b.

GONÇALVES, P. de S.; CARDINAL, A.B.B.; COSTA, R.B. da; BORTOLETTO, N.; GOUVÊA, L.R.L. Genetic variability and selection for laticiferous system characters in Hevea brasiliensis. Genetics and Molecular Biology, v.28, p.414-422, 2005.

GONÇALVES, P. de S.; FURTADO, E.L.; BATAGLIA, O.C.; ORTOLANI, A.A.; MAY, A.; BELLETTI, G.O. Genetics of anthracnose panel canker disease resistance and its relationship with yield and growth character in half-sib progenies of rubber tree (Hevea brasiliensis). Genetics and Molecular Biology, v.22, p.583-589, 1999.

GONÇALVES, P. de S.; MARTINS, A.L.M.; BORTOLETTO, N.; SAES, L.A. Selection and genetic gains for juvenile traits in progenies of Hevea in São Paulo State, Brazil. Genetics and Molecular Biology, v.27, p.758-764, 2004.

HERNANDEZ, F.B.T.; LEMOS FILHO, M.A.F.; BUZETTI, S. Software HIBRISA e o balanço hídrico de Ilha Solteira. Ilha Solteira: UNESP, 1995. 45p.

JAIN, S.M.; PRIYADARSHAN, P.M. Breeding plantation tree crops: tropical species. New York: Springer, 2009. 654p.

KAGEYAMA, P.Y.; VENCOVSKY, R. Variação genética em progênies de uma população de Eucalyptus grandis (Hill) Maiden. Revista IPEF, v.24, p.9-26, 1983.

MISSIO, R.F.; DIAS, L.A. dos S.; MORAES, M.L.T. de; RESENDE, M.D.V. de. Selection of Pinus caribaea var. bahamensis progênies based on the predicted genetic value. Crop Breeding and Applied Biotechnology, v.4, p.399-407, 2004.
MISSIO, R.F.; SILVA, A.M. da; DIAS, L.A. dos S.; MORAES, M.L.T. de; RESENDE, M.D.V. de. Estimates of genetic parameters and prediction of additive genetic values in Pinus kesya progenies. Crop Breeding and Applied Biotechnology, v.5, p.394-401, 2005.

MORETI, D.; GONÇALVES, P. de S.; GORGULHO, E.P.; MARTINS, A.L.M.; BORTOLETTO, N. Estimativas de parâmetros genéticos e ganhos esperados com a seleção de caracteres juvenis em progênies de seringueira. Pesquisa Agropecuária Brasileira, v.29, p.1099-1109, 1994.

PAIVA, J.R. de; RESENDE, M.D.V. de; CORDEIRO, E.R. Índice multiefeitos e estimativas de parâmetros genéticos em aceroleira. Pesquisa Agropecuária Brasileira, v.37, p.799-807, 2002.

PEDROZO, C.A.; BENITES, F.R.G.; BARBOSA, M.H.P.; RESENDE, M.D.V. de; SILVA, F.L. da. Eficiência de índices de seleção utilizando a metodologia REML/BLUP no melhoramento da cana-de-açúcar. Scientia Agraria, v.10, p.31-36, 2009.

RESENDE, M.D.V. de. Genética biométrica e estatística no melhoramento de plantas perenes. Brasília: Embrapa Informação Tecnológica; Colombo: Embrapa Florestas, 2002a. 975p.

RESENDE, M.D.V. de. Software Selegen - REML/BLUP. Colombo: Embrapa Florestas, 2002b. 67p. (Embrapa Florestas. Documentos, 77).

RESENDE, M.D.V. de; HIGA, A.R. Maximização da eficiência da seleção em testes de progênies de Eucalyptus através da utilização de todos os efeitos do modelo matemático. Boletim de Pesquisa Florestal, n.28/29, p.37-55, 1994.

SANTOS, H.G. dos; JACOMINE, P.K.T.; ANJOS, L.H.C. dos; OLIVEIRA, V.A. de; OLIVEIRA, J.B. de; COELHO, M.R.; LUMBRERAS, J.F.; CUNHA, T.J.F. (Ed.). Sistema brasileiro de classificação de solos. 2.ed. Rio de Janeiro: Embrapa Solos, 2006. 306p.

SOUZA, V.A.B. de; BYRNE, D.H.; TAYLOR, J.F. Predicted breeding values for nine plant and fruit characteristics of 28 peach genotypes. Journal of the American Society for Horticultural Science, v.125, p.460-465, 2000.

TAN, H.; SUBRAMANIAN, S.A. A five-parent diallel cross analysis for certain characters of young Hevea seedlings. In: INTERNACIONAL RUBBER CONFERENCE, 1975, Kuala Lumpur. Proceedings. Kuala Lumpur: RRIM, 1976. v.2, p.13-16.

VERARDI, C.K.; RESENDE, M.D.V. de; COSTA, R.B. da; GONÇALVES, P. de S. Adaptabilidade e estabilidade da produção de borracha e seleção em progênies de seringueira. Pesquisa Agropecuária Brasileira, v.44, p.1277-1282, 2009.

VIANA, J.M.S.; ALMEIDA, I.F. de; RESENDE, M.D.V. de; FARIA, V.R.; SILVA, F.F.E. BLUP for genetic evaluation of plants in non-inbred families of annual crops. Euphytica, v.174, p.31-39, 2010a.

VIANA, J.M.S.; SOBREIRA, F.M.; RESENDE, M.D.V. de; FARIA, V.R. Multi-trait BLUP in half-sib selection of annual crops. Plant Breeding, v.129, p.599-604, 2010b.

Recebido em 31 de agosto de 2010 e aprovado em 4 de novembro de 2010

Pesq. agropec. bras., Brasília, v.45, n.12, p.1419-1424, dez. 2010 